\title{
Genetic Diversity Analysis and In Silico Investigation of Post- Translational Modifications of Carboxypeptidase A1 (CpA1) in Sordaria fimicola
}

\author{
Uzma Naureen $^{1}$, Rabia Arif ${ }^{1}$, Faiza Akram ${ }^{1}$, Memuna Ghafoor Shahid ${ }^{\mathbf{2}}$ and Muhammad Saleem ${ }^{1}$ \\ ${ }^{1}$ Molecular Genetics Research Laboratory, Department of Botany, University of the Punjab, Lahore, Pakistan \\ ${ }^{2}$ Department of Botany, Government College University, Lahore, Pakistan \\ *For correspondence: phdgenetics@gmail.com \\ Received 11 May 2020; Accepted 31 October 2020; Published 10 January 2021
}

\begin{abstract}
Post-translational modifications (PTMs) regulate different complex mechanisms of cell and affect cell growth, stress, evolution of living organisms and adaptations due to environment. The purpose of the present research is to investigate the genetic diversity and PTMs of protease (Carboxypeptidase A1) n Sordaria fimicola. They perform a variety of functions ranging from housekeeping: e.g., protein maturation, signal peptide cleavage, signal transduction, intracellular protein turnover, immune response, apoptosis, and reproduction. S. fimicola is a microscopic filamentous fungus, has been preferably used in this study because of its easy growing pattern on Potato Dextrose Agar (PDA) and a short life cycle of 7 to 12 days. The genomic DNA of six of the strains $S$. fimicola was used to amplify the carboxypeptidases A1 gene $(C p A 1)$, the product size was $940 \mathrm{bp}$. The multiple sequence alignment of the nucleotide sequences of six strains of S. fimicola with Neurospora crassa (as a reference strain) was studied. The numbers of polymorphic sites in six strains of $S$. fimicola with respect to $N$. crassa were six. Posttranslational modifications were depicted by using bioinformatics tools i.e., YinOYang1.2, NetPhos 3.1 and NetNES 1.1 Server to calculate O-glycosylation, phosphorylation sites, and nuclear export signals respectively. The study has predicted 19 phosphorylation sites on serine residues for protease Carboxypeptidase A1 in S1 strains of S. fimicola while 15 phosphorylation sites on serine in N7 strain and 17 serine phosphorylation modifications were predicted in $N$. Crassa. The results of this research will be helpful for further in vitro investigations of this industrially important enzyme under study. (C) 2021 Friends Science Publishers
\end{abstract}

Keywords: Acetylation; Coprophilous fungus; Glycosylation; Phosphorylation; Protease; Strains

\section{Introduction}

Proteases account for $60 \%$ of total commercial enzyme market and the sources of proteases are microbes, fungi, animals, and plants (Boominadhan et al. 2009; Muszewskaet al. 2017). Microbial proteases are widely utilised in several industries such as brewing, detergent, leather, dairy, and food-processing factory (Arber 2000; Wood et al. 2011) The first aim of the current research is to explore genetic variations of protease Carboxypeptidase A1 (CpAl) of different strains of S. fimicola collected from the north-facing slope (NFS), south-facing slope (SFS) of "Evolution Canyon".

$S$. fimicola is a microscopic coprophilous fungus belongs to the class Sordariomycetes and is closely related to Neurospora and Podospora. It is found all over the world and produces black perithecia containing asci of eight dark ascospores in a linear arrangement. Due to having short life cycle of usually 7-12 days and easily grown in culture, $S$. fimicola is considered as a model organism for genetics study (Arif et al. 2019).

The natural selection of living organisms is competed for the adverse environmental conditions by genetic variations. Thus, evolution depends upon these variations because these are the causes of the evolutionary potential of organisms (Arif et al. 2017). Mutations are generated by these genetic variations and finally create frontier diversity of biomolecules like proteins by several PTMs. Living organisms preferred them to compete for the environmental stresses such as temperature, light, wind, water etc. The advancement of molecular biology quickly examines the exploration of the genetic biodiversity of different species. Bioinformatics and proteomics tools are predicting the details of modified sites of the molecules, which are used for joining and disjoining of functional groups (Marquez et al. 2018). This information of joining and disjoining of functional groups to the molecules is essential for the details of post-translational modifications network in the cell of living organisms (Shen 2013). Yu et al. (2007) reported that Ascomycota possesses more than two hundred types of

To cite this paper: Naureen U, R Arif, F Akram, MG Shahid, M Saleem (2021). Genetic diversity analysis and in silico investigation of post-translational modifications of carboxypeptidase A1 (CpA1) in Sordaria fimicola. Intl J Agric Biol 25:475-482 
PTMs. Marquez et al. (2018) and Jimenez-Morales et al. (2013) said that PTMs like acetylation, methylation, glycosylation, phosphorylation, S-nitrosylation and Ubiquitination commonly occur in eukaryotes (Chandramouli and Qian 2009).

This research aims to investigate the various PTMs of protease CpA1 using bioinformatics tools, which is a hot topic now a day because of their role in the understanding of different biological processes at the cellular level and designing of drugs against many diseases especially cancer (Chou 2019). This study has also been reported some particular protein kinases that are included in the phosphorylation of protease. Protein kinase $\mathrm{C}$ (PKC) accomplishes the role of controlling many proteins by adding the phosphate on the $-\mathrm{OH}$ (hydroxyl) groups of serine and threonine. This enzyme is activated in the increased concentration of diacylglycerol or calcium ions in the presence of signals such as an increase in the level of calcium ions or diacylglycerol (Khoury et al. 2011). The other protein kinase, CK2 (Casein kinase 2) is well known to occur in a physiological complex of tetramer (Sibanda et al. 2010). The DNA-Pkc is a threonine and serine protein kinase that consists of a single polypeptide chain made of 4128 amino acids (Turnham and Scott 2016).

To the best of our information, no scientific study was done on PTMs of protease CpA1 protein such as glycosylation, phosphorylation, and acetylation in N. crassa and S. fimicola. Regulation of protease Carboxypeptidase A1 protein by PTMs will characterize a new path of interest regarding the cell processes and cellular signalling and help in establishing the platform to produce proteases on small as well as on a large scale.

\section{Materials and Methods}

\section{Collection of samples and extraction of genomic DNA}

The samples of six parental strains obtained from the northfacing slope (NFS) and south-facing slope (SFS) of "Evolution Canyon", Israel, which possesses diverse environmental conditions. (These strains were received from Genetics department of Imperial College London). The sub-culturing of total six strains was done on PDA (potato dextrose agar) that is a nutrient medium for the growth of filamentous fungi followed by incubation at $20 \circ \mathrm{C}$ in an incubator. We obtained mature fungal growth in 8 days and then these sub-cultures were subjected for DNA extraction. DNA extraction was performed by adopting the method described by Pietro et al. (1995) and the DNA concentration, as well as quality, was assessed by calculating the absorbance at OD260/OD280.

\section{Amplification and sequencing}

Forward and reverse primers were designed through Primer 3 software for the amplification of CpA1 genes of all strains. The sequences of the forward primer were
ATCTTTCCTCACCGCC, and reverse primer was GTACTCGGCGACCATGGTAG. The PCR reaction volume was $15 \mu \mathrm{L}$, which contained $10 \mu \mathrm{L}$ PCR master mix (Gene All), $1 \mu \mathrm{L}$ forward primer, $1 \mu \mathrm{L}$ reverse primer and 3

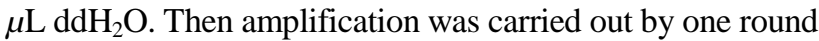
of amplification consists of initial denaturation at $95^{\circ} \mathrm{C}$ for $15 \mathrm{~min}$ followed by 30 cycles of denaturation at $95^{\circ} \mathrm{C}$ for 20 $\mathrm{s}$, annealing at $50^{\circ} \mathrm{C}$ for $40 \mathrm{sec}$ and extension at $72^{\circ} \mathrm{C}$ for 1 min, with a final extension step of $72^{\circ} \mathrm{C}$ for $5 \mathrm{~min}$. The amplification of PCR results was confirmed by running the product on gel electrophoreses on $0.8 \%$ agarose gel. The required bands were eluted and were sent for sequencing to Macrogen Korea.

\section{Prediction tools used for post-translational modifications}

PTMs were investigated with the help of these bioinformatics tools like YinOYang1.2, NetPhos 3.1 and NetNES 1.1 Servers. YinOYang 1.2 server is utilised to calculate glycosylation. NetPhos 3.1 server is used for phosphorylation sites prediction on residues of serine, threonine and tyrosine, whereas NetNES 1.1 server is utilised for nuclear export signals (NES). Online tool 'EMBOSS Transeq' was used to obtain the sequences of amino acid of amplified genes while the amino acid sequences of reference strain were retrieved from Uniprot.

\section{Homology modelling and model validation}

\section{Phyre2server}

(http://www.sbg.bio.ic.ac.uk/ phyre2/html/page.cgi?id=inde $\mathrm{x}$ ) is a reliable $3 \mathrm{D}$ structure prediction tool, which was used to build 3D models of protease with $100 \%$ confidence prediction. Afterward, the RAMPAGE tool was used to validate the $3 \mathrm{D}$ models available at http://mordred.bioc.cam.ac.uk/ rapper/rampage.php.

\section{Tertiary structure refinement}

Galaxy Refine (http://galaxy.seoklab.org/cgibin/submit.cgi?type=REFINE) tool was used to check the refinement of 3D structures of the template-based modelled protein. A unique web server uses a side chain algorithm with packaging and structural relaxation by molecular dynamics simulation. This tool increases the overall local and global quality of 3D structures. The tertiary structures of S1, N7 and N. crassa were subjected to the Galaxy Refine webserver to refine and enhance the quality of 3D models on mild and aggressive relaxation algorithm.

\section{Results}

The genomic DNA of all strains was used to amplify the protease CPAl gene and product size of 940 bp was obtained. Polymorphism study was carried out by aligning the nucleotide sequences of six strains of S. fimicola with a 
Genetic Analysis of Protease in Sordaria fimicola / Intl J Agric Biol, Vol 25, No 2, 2021

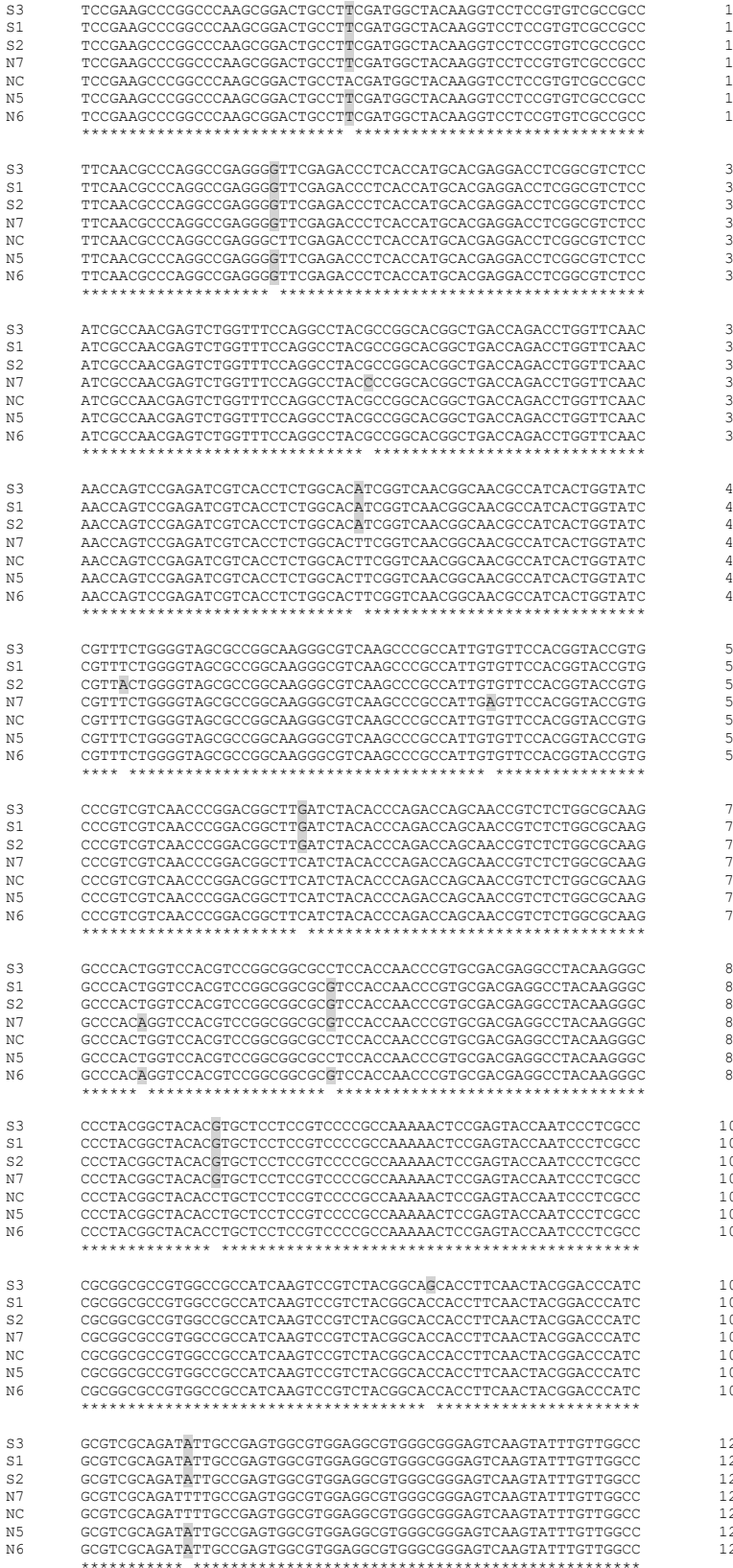

Fig. 1: Multiple sequence alignment of nucleotides sequence of protease $C p A 1$ regions of six $S$. fimicola strains with reference sequence of $N$. crassa. The gaps or spaces are showing polymorphic sites and symbols $(*)$ are showing similar or nonpolymorphic sites

reference organism $N$. crassa in clustal omega online tool. The numbers of polymorphic sites in the strains of $S$. fimicola compared to the $N$. crassa are 12 (Fig. 1). After sequencing, the sequences were subjected to blast tool at NCBI to check homologous sequences to those found for $S$. fimicola. BLAST used the $S$. fimicola sequence as a query sequence to find out the homologous region in $N$. crassa. The alignment of the amino acid sequences of six strains of

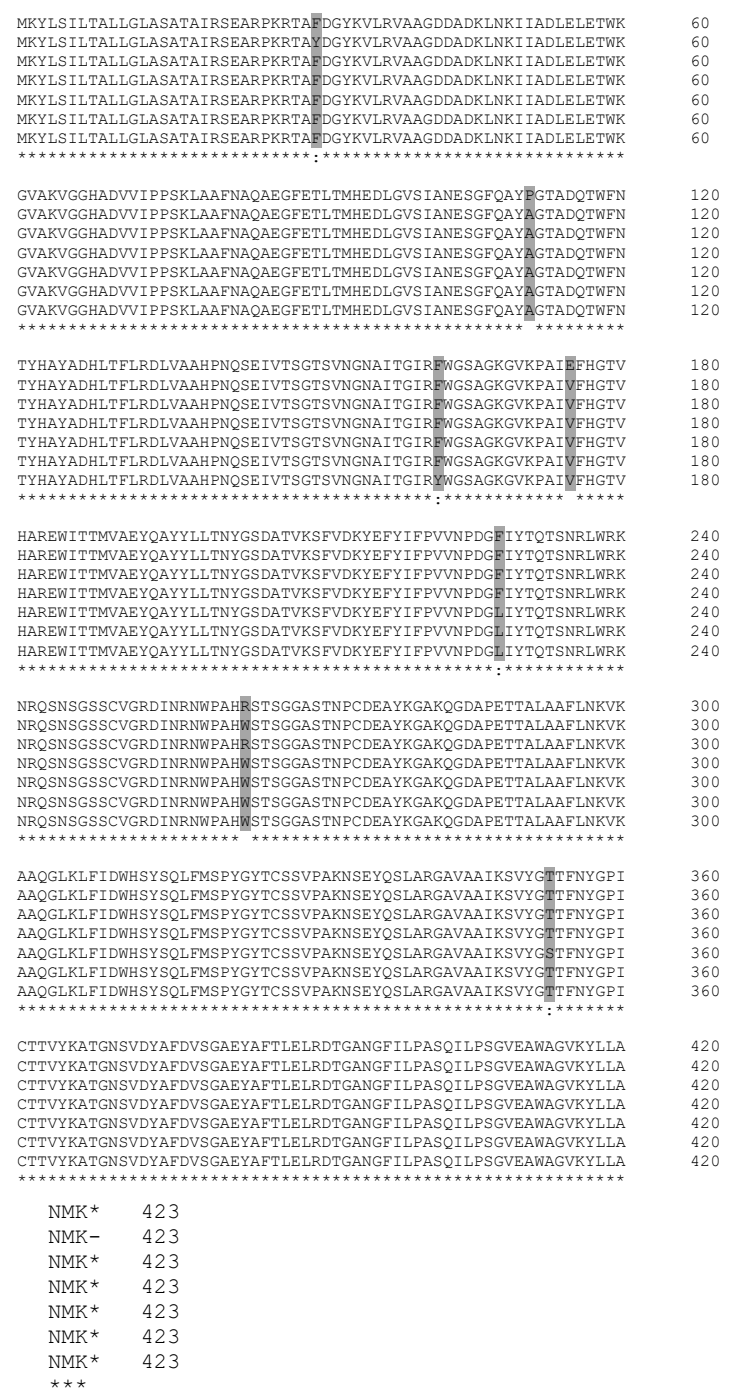

Fig. 2: Multiple sequence alignment of amino acid sequence of six strains of $S$. fimicola with reference sequence of $N$. crassa. The gaps are showing polymorphic sites and symbol (:) is showing the conservation among the species of strongly similar properties

S. fimicola with the reference sequence of $N$. crassa showed seven polymorphic sites, and four sites were found to be highly conserved among the species of strongly similar properties. The gaps indicate polymorphic sites and symbols (:) present the conservation among the species of significant features of similarities. The asterisks $(*)$ at the end of the amino acid sequence indicate the presence of stop codons (Fig. 2).

\section{O-glycosylation and YinOYang - predicted sites}

YinOYang and O-glycosylation sites at Serine, Threonine residues for CpA1 of $N$. crassa and $S$. fimicola were attained by YinOYang 1.2 (Table 1). In $N$. crassa, glycosylation was found on eight Serine residues, and five Threonine residues. SFS strains had nine serine, and four Threonine 
Table 1: Table is showing predicted O-glycosylation sites at Serine (S), Threonine (T) and Tyrosine (Y) residues as well as acetylation on Lysine (K) residues for carboxypeptidase A1 of $N$. crassa and S. fimicola. Glycosylation sites with asterisks are YinOYang sites, where interplay of phosphorylation and glycosylation is taking place.

\begin{tabular}{|c|c|c|c|}
\hline Organism & Amino Acid Residues & Glycosylation Positions & Acetylation on Lysine (K) \\
\hline & $\mathrm{S}$ & $200^{*}, 202,220,222 *, 283,284,290,335$ & \\
\hline \multirow{4}{*}{ N. crassa } & & Total $=08$ & $33,57,77,91,108,202,240,310,313,331,337,363,379,447,454$ \\
\hline & $\mathrm{T}$ & $103,221,245,281,348$ & Total $=14$ \\
\hline & & Total $=05$ & \\
\hline & $\mathrm{S}$ & $174^{*}, 184^{*}, 186,204,206^{*}, 267,268,274,319$ & \\
\hline \multirow[t]{2}{*}{ SFS Strains } & $\mathrm{T}$ & $\begin{array}{l}\text { Total }=09 \\
87,205,265,332 \\
\text { Total }=04\end{array}$ & $\begin{array}{l}2,26,34,46,60,77,171,209,279,282,300,306,332,348,416,423 \\
\text { Total }=16\end{array}$ \\
\hline & $\mathrm{S}$ & $184^{*}, 186,204,206 *, 267,268,274,319$ & \\
\hline NFS Strains & $\mathrm{T}$ & $\begin{array}{l}\text { Total }=08 \\
53,87,205^{*}, 229,265,332 \\
\text { Total }=06\end{array}$ & $\begin{array}{l}2,26,34,46,60,77,171,209,279,282,300,306,332,348,416,423 \\
\text { Total }=16\end{array}$ \\
\hline
\end{tabular}

(a)

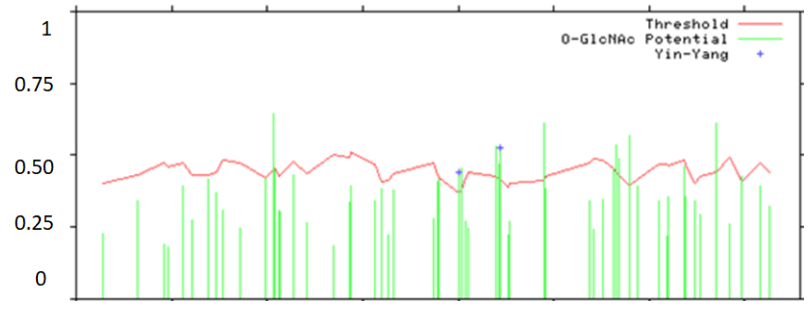

(b)

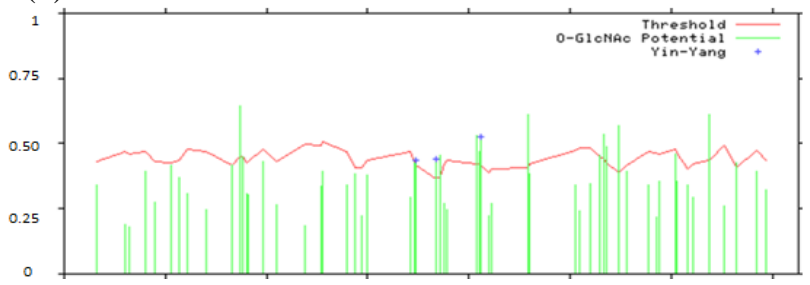

(c)

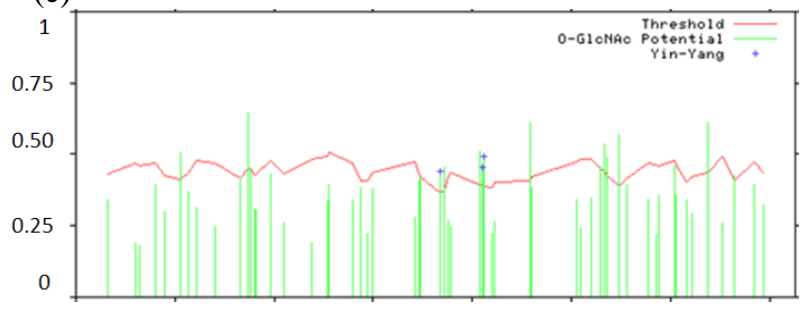

Fig. 3: Graphs are showing glycosylation potential of each $\mathrm{O}$ GlcNAc (O-linked acetyl glucosamine) modified sites (a) $N$. crassa (b) SFS strains and (c) NFS strains of $S$. fimicola. Vertical lines in green color are showing O-GlcNAc potential, red horizontal line is showing threshold level $(0.5)$ and blue plus (+) signs are representing YinOYang sites

glycosylation modifications; while NFS strains have eight serine glycosylation modifications and six threonine modifications. The residues with asterisks are YinOYang sites where the interchange of phosphorylation and glycosylation is taking place. The potential of all glycosylation sites is shown in Fig. 3.

\section{Prediction of acetylation, phosphorylation and nuclear export signals (NES)}

Predicted sites of acetylation of internal lysine residues for Protease CpA1of S. fimicola and N. crassa are shown in Table 1 . We found 14 acetylation sites in N. crassa and 16 in each of SFS and NFS strains. All possible phosphorylation sites of $N$. crassa and $S$. fimicola are given in Table 2. The sites (S-407, T-58, T-231, T-325, T-353, and T-363) of $S$. fimicola are different from $N$. crassa due to the genetic variation after PTMs. The sites (S-403, T- 266, T-279, Y-58, Y-230 and Y-231) of $N$. crassa are different from $S$. fimicola due to genetic variations. Nuclear export signals on residue 56-L (Lucien) and 93-M (Methionine) in N. crassa and $S$. fimicola have been predicted as shown in Fig. 4.

\section{Molecular modelling and structure validation}

All of the three proteins of N. crassa, Sordaria fimicola N7, and Sordaria fimicola S1 were modelled using the Phyre2 structure prediction server. The template used to model the N7, S1, and N. crassa proteins were the human CpA1 (PDB ID: 5OM9). Based on this template, all amino acid residues of input protein sequences were modelled as one domain. The overall uGDT (un-normalized global distance test) of $S$. fimicola N7 was 317 (74), S. fimicola S1 was 316(74), and $N$. crassa protein was 319 (75), presenting the same residues number in the alignment. A total of 432 amino acid residues were modelled as a single domain with an $8 \%$ disorder. Each model has its own dimensions ( $⿱$ ) with small differences; (a) X: 50.735, Y: 50.990, Z: 60.736 (b) X: 50.716, Y: 55.215, Z: 60.736 (c) X: 50.728, Y: 51.564, Z: 60.73 (Fig. 5).

Secondary structure information revealed the presence of $35 \%$ helix, $15 \%$ Beta sheet, and $49 \%$ coiled structure. The P-value of the 3D model suggests the relative quality of the predicted model, lesser the P-value, excellent the quality of the model. The P-value got for the predicted N7 model was $2.40 \mathrm{e}^{-14}, 3.35 \mathrm{e}^{-14}$ for $\mathrm{S} 1$ and $4.56 \mathrm{e}-14$ for $\mathrm{N}$. crassa expressing the excellent quality of the model. Galaxy Refine 
Table 2: Phosphorylation predicted sites with their protein kinases for Carboxypeptidase A1 protein of $N$. crassa and different strains of $S$. fimicola. Numbers in third column are showing the phosphorylation positions on serine, threonine and tyrosine residues of Carboxypeptidase A1. The numbers in the others columns (last six) are showing the positions, where the specific protein kinase involved in the phosphorylation of its respective residue i.e., serine, threonine, and tyrosine

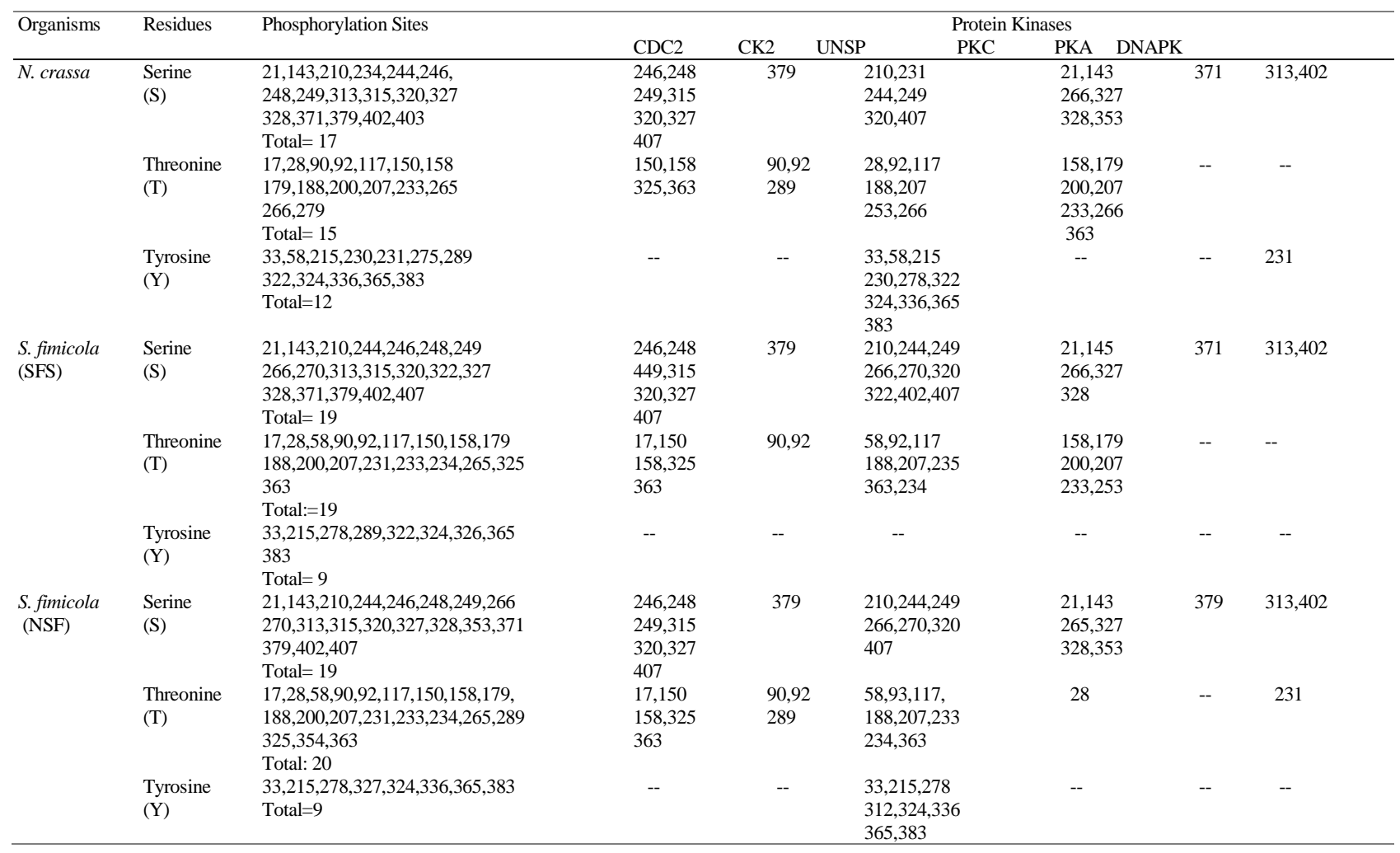

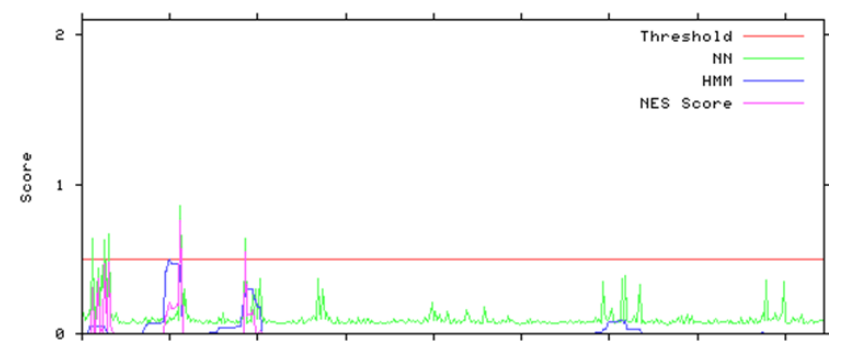

Fig. 4: Graphical representation of leucine rich nuclear export signals (NES) potential for of $N$. crassa and six S. fimicola strains. Green peaks are showing NN signals, blue peaks are showing HMM signals, purple peaks are showing NES signals and red horizontal line is presenting threshold level, which is 0.5 and above Key: X-axis showing sequence position; Y-axis showing O-Glycosylation potential

showed that the number of residues increased in the favoured region. After the refinement of N7 model, 95.5\% residues were present in the favoured region, $3.6 \%$ residues in the allowed region and only $1 \%$ of residues were in the outlier region. S1 model refinement results presented $96.2 \%$ residues in the favoured region, $3.1 \%$ residues in the allowed region and only $0.7 \%$ of residues were in the outlier region. Likewise, the N. crassa protein model has $97.1 \%$ residues in the favoured region, while $2.4 \%$ residues in the allowed region and only $0.5 \%$ residues were present in the outlier region (Fig. 6). These refinement results show the reliability of the Phyre2 3D model prediction tool and the validity of prediction.

\section{Discussion}

The protease $\mathrm{CpA}$ lis first time reported in S. fimicola. Genetic variations were studied in the protease CpA1 gene of S. fimicola. Our study has sharply linked the genetic diversity of CPAl with PTMs of protease CpA1 in $S$. fimicola. We had observed more polymorphic sites in the SFS strains than in the NFS strains. Other co-workers have also been found more polymorphism in the SFS strains as compared to the NFS strains of $S$. fimicola in their studies (Saleem et al. 2001; Ishfaq et al. 2014; Arif et al. 2017; Bukhari et al. 2020; Mobeen et al. 2020). Due to the harsh and xeric environmental conditions at SFS slope, the strains of this slope bear more polymorphism than the strains of NFS slope (having mild conditions) of "Evolution Canyon".

Walsh et al. (2005) said that PTMs are referred to as biochemical processes that take place after its synthesis. This study has predicted four types of PTMs; phosphorylation, O-glycosylation, acetylation and nuclear export signals (NES). The phosphorylation process takes 


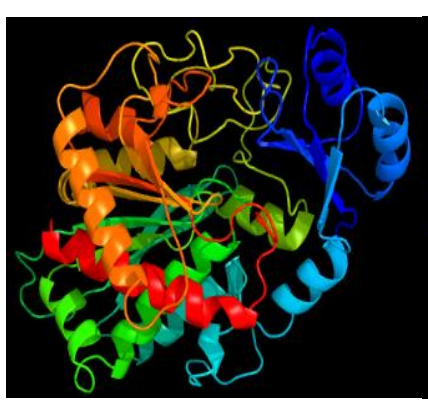

(a)

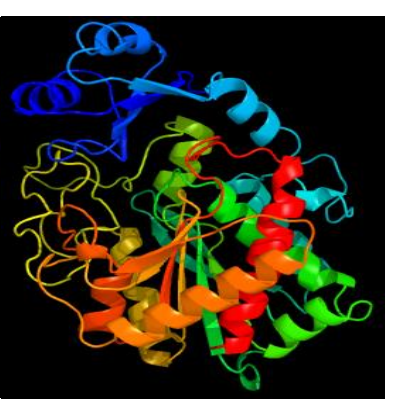

(b)

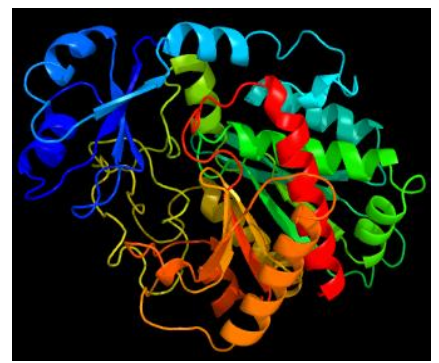

(c)

Fig. 5: 3D structure of (a) N. crassa (b) S1 and (c) N7 strains with $100 \%$ confidence prediction by Phyre2. Arrows in the structure are showing $\beta$-sheets, coiled ribbons are $\alpha$-helix and sticks are coils. Each model has its own dimensions ( $)$ with small differences; (a) X: 50.735, Y: 50.990, Z: 60.736 (b) X: 50.716, Y: $55.215, \mathrm{Z}: 60.736$ (c) X: 50.728, Y: 51.564, Z: 60.73

place at specific residues of serine and threonine. It has effects on structural and signalling of the cell, whereas the percentage of phosphorylation at residues of Tyrosine is only $1 \%$, which is linked in the cell signalling (Ishfaq et al. 2017). We predicted 19 phosphorylation sites on serine residues for $\mathrm{CpA} 1$ in SFS strains of $S$. fimicola. In comparison, 15 phosphorylation sites on serine in NFS strains and 17 serine phosphorylation modifications were predicted in $N$. crassa (Table 2).

The present study has found phosphorylation on Ser143; Ser-248 in N. crassa and as well as in S. fimicola (NFS $\&$ SFS strains), so these are considered to be highly conserved in them. In most of the eukaryotes from fungi to mammals, Ser-248 and Thr-233 were found to play a conserved task in controlling the development of cells (Horn et al. 2009). Huang et al. (2012) have been found phosphorylation on these sites experimentally.

During the present investigation, we have found that serine and threonine phosphatases are actively involved in the phosphorylation of protease CpA1 of S. fimicola and $N$. crassa. CDC2, CK2, UNSP, PKC, PKA, DNA-PK are found to be highly engaged in phosphorylation of CpA1 of $N$. crassa and S. fimicola (Table 2). The role of protein kinases is very vital in phosphorylation. Their function is to transfer a phosphate group from adenosine triphosphate to the protein substrate and changed it into phosphorylated. PKC and PKA kinases in fungi perform many essential features like regulation of cell, growth, synthesis of protein, (a)

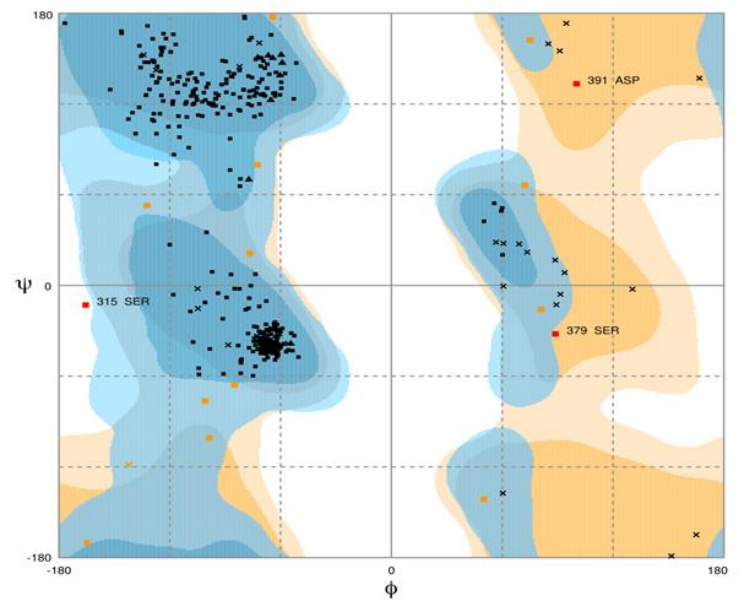

(b)

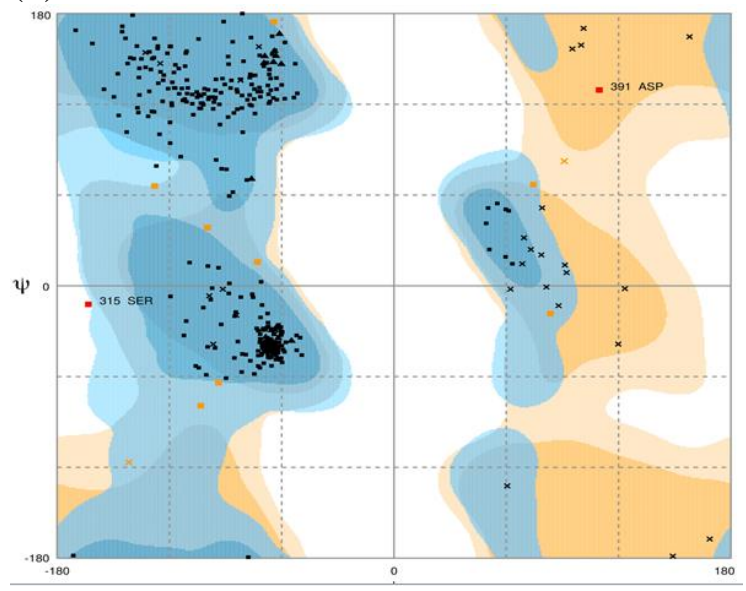

(c)
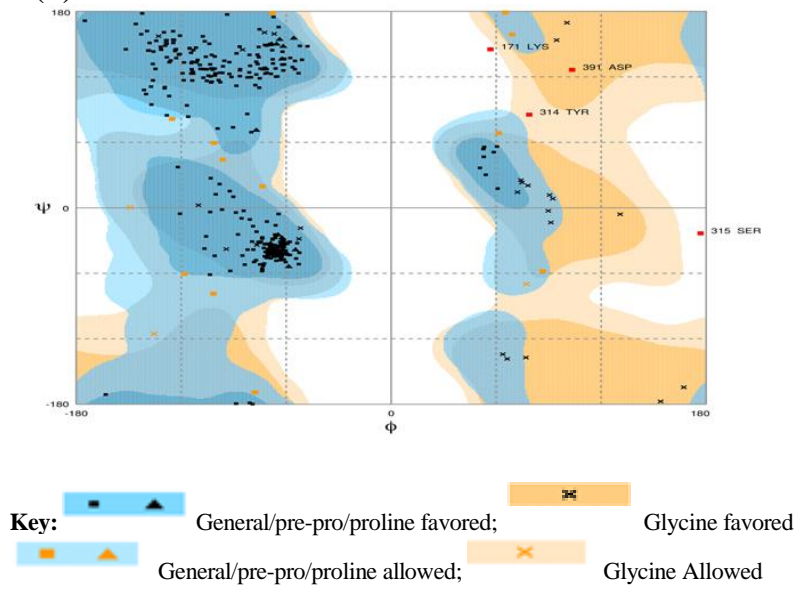

Fig. 6: These graphs are showing the refinement results of 3D models of (a) N. crassa (b) S1 (c) and N7 strains of S. fimicola for protease CpA1 using Galaxy Refine server to check the validity and reliability of 3D models

and maintain cell integrity (Albataineh et al. 2014). A current BLAST search has also shown the occurrence of homologs for numerous significant kinases (PKA, Cek1- 
MAPK, PKC,) and enzymes like phosphatases are expected to play roles in pathogenicity (Leach and Brown 2012). Protein kinases (PKC, CDC2, UNSP, and PKA) involved in phosphorylation of COX1 (Cytochrome c oxidase) reported by (Arif et al. 2019).

O-glycosylation is another alterable type of modification, which is responsible for immunity, survival, signalling and transcription (Zhang et al. 2011). We have found 13 and $14 \mathrm{O}$-glycosylation sites for proteases CpA1in SFS and NFS strains, respectively. Some differences in the O-GlcNAc (O-linked acetyl glucosamine) modified sites among strains are found i.e., NFS strains have two novel sites (T-53 and T-229) that are absent in SFS strains, likewise S-174* is not present in the NFS strains. These differences are the reflections of polymorphism. Some sites (T-205*, S-174*, S-184*, T-205*, S-206*) have shown interplay between glycosylation and phosphorylation (Table 1). Jamil et al. (2018) have been reported interplay between O-glycosylation and phosphorylation at six serine and threonine residues for Histone $\mathrm{H} 3$ of $S$. fimicola using YinOYang server-a reliable tool for the prediction of protein O-glycosylation.

The acetylation is a process of transfer of an acetyl group $\left(\mathrm{CH}_{3} \mathrm{CO}\right)$ to other molecules. The acetylation is a modification that affects the function of a protein by changing its properties such as solubility, hydrophobicity, and properties of the surface. All these changes can affect protein conformation and interactions with substrates, cofactors, and other macromolecules (Christensen et al. 2019). We have observed 14 acetylation modifications in $N$. crassa and 16 similar acetylation modifications on internal lysine $(\mathrm{K})$ in all strains of S. fimicola (Table 1). Carabetta et al. (2016) had reported the acetylation a K240 in Bacillus subtilis, which reduces cell length, width, and peptidoglycan thickness. This study has also been reported K-240 modification, which might perform the same functions as have been reported in B. subtilis by Carabetta et al. (2016).

Nuclear export signals are exceedingly essential elements for the biomolecules because these signals regulate the subcellular localization of these molecules. These signals are responsible for the export of proteins and transcriptional factors from the nucleus to the cytoplasm (Fischer et al. 1995). Nuclear export signals (NES) on residue 56-L (Leucine) and 93-M (Methionine) in N. crassa and $S$. fimicola have been predicted are shown in Fig. 4. The existence of NES in protease CpA1 in N. crassa and all strains of $S$. fimicola evidenced that these nuclear export signals have their role in the regulation of this protein.

\section{Conclusion}

The SFS strains of have more tendency of genetic variation than NFS strains due to the stressful conditions of southfacing slope. These variations on the $\mathrm{CpA} 1$ region might be helpful in the survival under stressful conditions by producing diverse protein motifs through various posttranslational modifications. Although the molecular basis of these genetic variations has been investigated in this study, but the functional study of each polymorphic site is required to device the specific functions related to these sites.

\section{Author Contributions}

Uzma Naureen perform the major experiments Rabia Arif and Muhammad Saleem Plan the research work Faiza Akram and Memuna Ghafoor Shahid help in manuscript write up.

\section{References}

Albataineh MT, A Lazzell, JL Lopez-Ribot, D Kadosh (2014). Ppg1, a PP2A-type protein phosphatase, controls filament extension and virulence in Candida albicans. Eukaryot Cell 13:1538-1547

Arber W (2000). Genetic variation: Molecular mechanisms and impact on microbial evolution. FEMS Microbiol Rev 24:1-7

Arif R, SH Bukhari, M Ishfaq, MG Shahid, SF Lee, M Saleem (2019). Genetic variation and post-translational modifications of cytochrome c oxidase-1 (COX1) in different strains of Sordaria fimicola. Intl J Agric Biol 21:1055-1062

Arif R, F Akram, T Jamil, H Mukhtar, SF Lee, M Saleem (2017). Genetic variation and its reflection on posttranslational modifications in frequency clock and mating type a-1 proteins in Sordaria fimicola. Biol Med Res Intl 2017; Article 1268623

Boominadhan U, R Rajakumar, PKV Sivakumaar, MM Joe (2009). Optimization of protease enzyme production using Bacillus spp. isolated from different wastes. Bot Res Intl 2:83-87

Bukhari SH, I Mobeen, U Naureen, F Akram, R Arif, MG Shahid, M Saleem (2020). Analysis of genetic polymorphisms and posttranslational modifications of cytochrome C-1 in Sordaria fimicola. Intl J Agric Biol 23:675-680

Carabetta VJ, TM Greco, AW Tanner, IM Cristea, D Dubnau (2016) Temporal regulation of the Bacillus subtilis acetylome and evidence for a role of Mre B acetylation in cell wall growth. mSystems 1; Article e00005-16

Chandramouli K, P Qian (2009). Proteomics: Challenges, techniques and possibilities to overcome biological sample complexity. Hum Genom Proteom 2009:1-22

Chou KC (2019). Progresses in predicting post-translational modification. Intl J Pept Res Ther 26:873-888

Christensen DG, X Xie, N Basisty, J Byrnes, SM Sweeney, B Schilling, A. Wolf (2019). Post-translational protein acetylation: An elegant mechanism for bacteria to dynamically regulate metabolic functions. Front Microbiol 10; Article 1604

Fischer U, J Huber, WC Boelens, IW Mattaj, R Luhrmann (1995). Cell $82: 475-483$

Horn DL, EJD Neofytos, JA Anaissie, WJ Fishman, AJ Steinbach, KA Olyaei, MA Marr, CH Pfaller, KM Chan, KM Webster (2009). Epidemiology and outcomes of candidemia in 2019 patients: Data from the prospective antifungal therapy alliance registry. Clin Infect Dis 48:1695-1703

Huang OW, X Ma, J Yin, J Flinders, T Maurer, N Kayagaki, Q Phung, I Bosanac, D Arnott, VM Dixit, SG Hymowitz (2012). Phosphorylation-dependent activity of the deubiquitinase DUBA. Nat Struct Mol Biol 19:171-175

Ishfaq M, N Mahmood, IA Nasir, M Saleem (2017). Biochemical and molecular analysis of superoxide dismutase in Sordaria fimicola and Aspergillus niger collected from different environments. Pol $J$ Environ Stud 26:115-125

Ishfaq M, N Mahmood, IA Nasir, M Saleem (2014). Molecular and biochemical screening of local Aspergillus niger strains efficient in catalase and laccase enzyme production. Intl J Agric Biol 16:177-182 
Jamil T, N Sami, R Arif, Q Rashid, M Saleem (2018). H3/H4 Histone genes variations and its effect on posttranslational modifications in various strains of Sordaria fimicola. Intl J Agric Biol 20:1021-1026

Jimenez-Morales D, L Adamian, D Shi, J Liang (2013). Lysine carboxylation: Unveiling a spontaneous post-translational modification. Acta Crystallogr D Biol Crystallogr 70:48-57

Khoury GA, RC Baliban, CA Floudas (2011). Proteome-wide posttranslational modification statistics: Frequency analysis and curation of the swiss-prot database. Sci Rep 1; Article 90

Leach MD, AJ Brown (2012). Posttranslational modifications of proteins in the pathobiology of medically relevant fungi. Eukaryot Cell 11:98-108

Marquez J, SR Lee, N Kim, J Han (2018). Post-translational modifications of cardiac mitochondrial proteins in cardiovascular disease not lost in translation. Kor Circ J 46:1-12

Mobeen I, R Arif, A Rasheed, F Akram, MG Shahid, M Saleem (2020). Genetic and post-translational modification analysis of translational associated protein RKM4 in Sordaria fimicola. Intl J Agric Biol 23:935-942

MuszewskaA, MM Stepniewska-Dziubinska, K Steczkiewicz, J Pawlowska, A Dziedzic, K Ginalski (2017). Fungal lifestyle reflected in serine protease repertoire. Sci Rep 7; Article 9147

Pietro S, TM Fulton, J Chunwongesm, SD Tanksley (1995). Extraction of high-quality DNA for genome sequencing. Mol Biol Rep 13:207
Saleem M, BC Lamb, E Nevo (2001). Inherited differences in crossing over and gene conversion frequencies between wild strains of Sordaria fimicola from "Evolution Canyon". Genetics 159:1573-1593

Shen B (2013). Bioinformatics for Diagnosis, Prognosis and Treatment of Complex Diseases, Vol. 4. Springer Science + Business Media, Berlin, Germany

Sibanda BL, DY Chirgadze, TLBlundell (2010). Crystal structure of DNAPKcs reveals a large open-ring cradle comprised of HEAT repeats. Nature 463:118-121

Turnham RE, JD Scott (2016). Protein kinase A catalytic subunit isoform PRKACA; History, function and physiology. Gene 577:101-108

Walsh CT, S Garneau-Tsodikova, GJ Gatto (2005). Protein posttranslational modifications: The chemistry of proteome diversifications. Angew Chem Intl 44:7342-7372

Wood JR, JM Wilmshurst, TH Worthy, A Cooper (2011). Sporormiella as a proxy for non-mammalian herbivores in island ecosystems. Quat $S c i$ Rev 30:915-920

Yu LR, HJ Issaq, TD Veenstra (2007). Phosphoproteomics for the discovery of kinases as cancer biomarkers and drug targets. Proteomics 1:1042-1057

Zhang S, K Roche, HP Nasheuer, NF Lowndes (2011). Modification of histones by sugar $\beta$-N-Acetylglucosamine (GlcNAc) occurs on multiple residues, including histone $\mathrm{H} 3$ Serine 10 , and is cell cycleregulated. J Biol Chem 286:37483-37495 